\title{
Papers vanish in mis-citation black hole
}

Sir — The assessment of research quality is increasingly based on impact factors and citation analyses of published work. But there are possible biases in these measures $^{1-4}$. Is the fact that Italian, French, German and Japanese publications received a less than average share of citations due to a lack of quality ${ }^{3}$ or to systematic bias ${ }^{1}$ ?

Further analysis of the data in ref. 1 on papers by Italian scientists reveals a strong positive relationship between journal impact factor class (IFC) and proportion of publications undercited (Fig. 1). Furthermore, the proportion of publications undercited in the highest IFC is significantly greater than the corresponding proportion in the lowest IFC $\left(\chi_{1}^{2}=4.89, P=0.027\right)$. These effects strengthen the negative implications of underciting for Italian scientists: not only is their work undercited in general but, as the quality of their work increases, it is less recognized! These results clearly support Paris et al.'s conclusions ${ }^{1}$ that undercitation does not result from substandard publications ${ }^{3}$.

What drives the undercitation? I suggest that incorrect citation may be one factor. Price $^{2}$ has analysed the citations of three highly cited publications, with astonishing

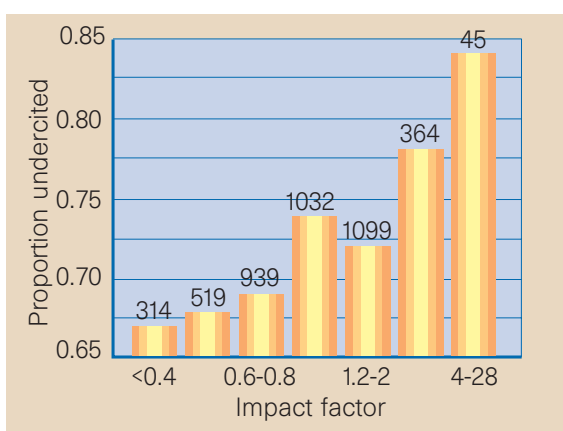

Figure 1 Relationship between the proportion of Italian publications undercited and the journal impact factor class (Spearman's $r=0.964, N=7$, $P<0.001)$. The numbers above the bars indicate the number of publications in each class (data from ref. 1).

results: the publications were cited incorrectly in more than 200 different ways. An analysis of the data for the G7 group of countries from Table 1 in ref. 3 reveals that all non-English-speaking countries receive less than their share of citations for their share of publications, whereas the English-speaking countries all receive an equal or greater share of citations for their share of publications. This difference is statistically significant
(Fisher's exact $P=0.029$ ). Given the high incidence of incorrect citations overall ${ }^{2}$, there may also be a general tendency towards making more mistakes when handling citations with unfamiliar names from another language.

When this tendency is coupled with the fact that almost half of all scientific publications come from English-speaking countries $^{3}$, the hazard of incorrect citation may not be random, but instead biased towards non-English-speaking countries. Because incorrect citations do not appear in citation indexes ${ }^{2}$, undercitation may be a result of incorrect citation rather than an indicator of poor-quality research in non-English-speaking countries.

Nevertheless, the reason behind the positive relationship between impact factor and undercitation (Fig.1) remains a mystery that urgently requires further attention.

\section{Janne S. Kotiaho}

Department of Zoology,

University of Western Australia,

Nedlands, WA 6009, Australia

1. Paris, G., De Leo, G., Menozzi, P. \& Gatto, M. Nature 396, 210 (1998)

2. Price, N. C. Nature 395, 538 (1998).

3. May, R. M. Science 275, 793-796 (1997).

4. Barreto, G. R. Science 276, 882-883 (1997).

\section{Engineering a}

\section{longer life}

Sir - In the excellent article "Eighteenninety-nine and all that", Isambard Kingdom Brunel is said to have died in 1869 (Nature 397, 15-18; 1999). Would that he had! We would have had ten years more of one of the world's greatest engineers.

\section{Nigel L. Firth}

Nova Scotia Agricultural College,

Truro, Nova Scotia B2N 5E3, Canada

\section{Managing $\mathrm{SOHO}$}

\section{Sir - Tony Reichhardt's News article}

"Rescued satellite to get more managers" is almost entirely accurate in its explanation of the concerns of operating a complex spacecraft during a period of pressure to reduce the costs of flight operations (Nature 396, 399; 1998). But it is misleading in stating that "Management of the $\$ 1$ billion satellite was to have merged with that of the other projects in the ... ISTP programme to save money".
In fact, it was the operation of SOHO and the ISTP WIND and POLAR satellites that was to have been merged in order to reduce costs, and indeed this is still going ahead. The management of SOHO flight operations, resources allocation and scientific investigations was never planned to be merged with the rest of ISTP. Indeed, the plan was to continue the already existing, shared management of $\mathrm{SOHO}$, WIND, POLAR and several other space science assets operated at NASA Goddard.

I believe the new $\mathrm{SOHO}$ programme office offers an opportunity to manage a major space science asset in a manner consistent with both its scientific importance and the level of operational risk.

Joseph B. Gurman

NASA Goddard Space Flight Center, Code 682, Greenbelt, Maryland 20771, USA

\section{Controversy over the cloning of mice}

Sir — Tsunoda and Kato ${ }^{1}$ report the successful cloning of mice after transferring cell nuclei from late preimplantation embryos. Our earlier studies ${ }^{2}$, using different methods, were mostly neglected or rejected. But Tsunoda and Kato found similar, although not identical, results concerning the ability of nuclei from the inner cell mass (ICM) of blastocysts to give rise to adult mice.

It is important to note that, under different experimental conditions, similar results have confirmed the developmental potential of ICM nuclei. Tsunoda and Kato also report the successful cloning of mice after transferring cell nuclei from the trophoblast of blastocysts, a type of cell thought to be specialized for its extraembryonic development.

In contrast, McGrath and Solter ${ }^{3}$ concluded from negative results that the cloning of mice using donor nuclei from late preimplantation embryos was impossible, and suggested that the "inability of cell nuclei from these stages to support development reflects rapid loss of totipotency of the transferred nucleus".

Now, with these positive data on the cloning of mice, the time has come for the correct evaluation of our earlier results on the first cloning of a mammal.

It has been argued that the early activation of the mouse embryonic genome is a barrier to cloning, leaving too little time 
for the nucleus to be reprogrammed. But even Solter ${ }^{4}$ now admits that this is not insurmountable, in the light of successful experiments by Wakayama et al. ${ }^{5}$ using adult cumulus cells for the cloning of mice.

\section{Karl Illmensee}

Universitatsklinik fur Frauenheilkunde,

Anichstrasse 35, A-6020 Innsbruck, Austria

1. Tsunoda, Y. \& Kato, Y. J. Reprod. Fertil. 113, 181-184 (1998).

2. Illmensee, K. \& Hoppe, P. Cell 23, 9-18 (1981).

3. McGrath, J. \& Solter, D. Science 226, 1317-1319 (1984).

4. Solter, D. Nature 394, 315-316 (1998).

5. Wakayama, T., Perry, A. C. F., Zuccotti, M., Johnson, K. R. \& Yanagimachi, R. Nature 394, 369-374 (1998).

\section{Forging links in an}

\section{electronic paper chain}

Sir — The Briefing on electronic journals was interesting and timely (Nature 397, 195-200; 1999). But one issue that was not addressed was how Internet publishing will change the style of scientific writing. One imagines that the length of on-line articles will be less restricted than paper ones even in the most selective journals. This will encourage a more thorough, but perhaps windier, writing style.

Counterbalancing this are the possibilities of hypertext. This will allow authors to connect their articles to supplementary material on their own sites or in external databases. This will enable them to reduce the main body of their text and to make it less technical, moving the details to linked sections. It may also lead to a more segmented, 'fact-box' style of presentation. Copious links will require careful layout, ensuring that they remain stable and reflect an underlying logic.

Finally, the use of hypertext in papers raises the issue of whether authors will be free to modify linked material on their own websites, or whether the content related to a paper should be frozen on submission. This is especially relevant to the refereeing process.

\section{Mark Gerstein}

Department of Molecular Biophysics and Biochemistry, Yale University, Bass 432A, 266 Whitney Avenue, New Haven, Connecticut 06520-8114, USA

\section{The editor as an endangered species}

Sir - I used to feel a great sense of security in my job as editor of Physical Review Letters. Receipts continue to increase, the journals of the American Physical Society are leading in most aspects of electronic publishing, and, of course, an editor could never be replaced by a computer program.

Alas, your Briefing on electronic journals tells me I am an endangered species (Nature 397, 195-200; 1999). Apparently the Journal of High Energy Physics already has a robot that reads manuscripts and assigns them to referees. I imagine your reporter meant to say "assigns them to editors". And, according to a picture in your article, my boss Martin Blume has become a web page! He was spotted in the editor-in-chief's office recently, so that must have been a printer's error.

No doubt the electronic future will have robots that will avoid such errors, but will readers be able to trust them?

Please tell me that editors are really needed.

\section{Gene L. Wells}

Physical Review Letters,

1 Research Road, Box 9000, Ridge,

New York 11961-9000, USA

\section{Space-grown crystals may prove their worth}

Sir - The first building block of the International Space Station was launched on 20 November 1998, but the potential uses of the space station are still under debate. A recommendation to scrap NASA's research on protein crystals was reported recently ${ }^{1}$. The reason given was that no serious contributions to our knowledge of protein structure have yet been made in space. We wish to point out, on the basis of recent experimental and theoretical evidence, that in many cases the potential benefits of the microgravity environment have not been fully exploited. This explains the low rate of success of protein crystallization in microgravity and opens up the scope for enhancing the efficiency of experimentation in space.

Microgravity eliminates sedimentation and convective mixing, so offering a more homogeneous growth medium compared with growth on Earth. Since this is likely to improve the degree of perfection of the crystals, why has microgravity crystallization not been more successful?

There are four common methods for crystallizing proteins: batch, vapour diffusion, dialysis and free interface diffusion (FID). Vapour diffusion is the most successful technique for crystallization on Earth. Naturally it became the method of choice for crystallization in microgravity. Thanks to the European Space Agency providing new means of conducting experiments in a far more systematic way, a comparison of microgravity crystallization using different methods was facilitated. The results demonstrated that vapour diffusion is not the best technique for crystallization in microgravity ${ }^{2}$.

Images from CCD cameras recorded during flights showed that some crystals grown by vapour diffusion displayed a cyclic motion within the aqueous drop in which they grow ${ }^{3}$. This motion is attributed to Marangoni convection, an effect which serves to reduce concentration gradients along the interface between the solution and the vapour ${ }^{4}$. In the case of FID and dialysis there is no interface between solution and vapour and this cyclic motion does not occur ${ }^{5}$.

Cyclic movement of the crystals in microgravity destroys the very benefit that is sought from the unique environment of outer space and thus may be a limiting factor in the ultimate perfection (indicated by X-ray diffraction) of the crystals that can be obtained ${ }^{6-8}$.

Several researchers have mentioned that crystals grown in microgravity by dialysis and FID methods appeared to be superior to those grown by vapour diffusion ${ }^{9,10}$, but those results were not taken seriously enough and most experiments were still done by vapour diffusion. Recent video recordings ${ }^{2,3,5}$ show beyond any doubt that crystal movement (akin to sedimentation referred to above) takes place in the case of vapour diffusion but not with the other methods.

It is apparent that we may have only now grasped how best to use the microgravity environment. Hence it would be a great shame if the experiments were scrapped now, just at the stage when a better understanding of crystallization in space and its fluid physics and biophysical chemistry is being gained. We are now in a position to explore more efficient ways of increasing the success rate of these experiments. The translation of the results to improve protein structure determination will come later. The stage of basic research is not yet completed to allow targeted exploitation to take place.

\section{Naomi E. Chayen}

Physics Department, Imperial College of Science, Technology and Medicine, London SW7 2BZ, UK

\section{John R. Helliwell}

Department of Chemistry, University of Manchester Manchester M13 9PL, UK

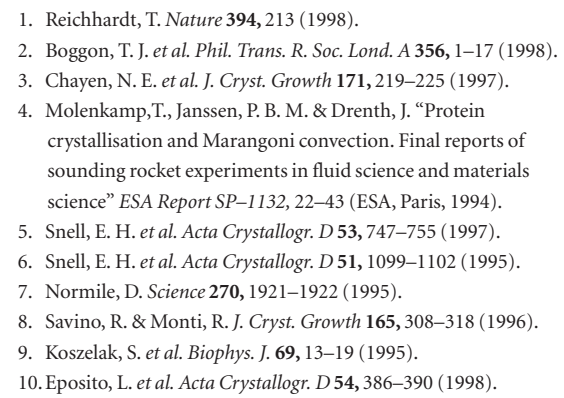

